\title{
Using noncontrast cardiac CT and coronary artery calcification measurements for cardiovascular risk assessment and management in asymptomatic adults
}

\author{
This article was published in the following Dove Press journal: \\ Vascular Health and Risk Management \\ 6 July 2010 \\ Number of times this article has been viewed
}

\author{
John A Rumberger \\ The Mayo Clinic and Foundation \\ Director of Cardiac Imaging \\ The Princeton Longevity Center, \\ Princeton, NJ, USA
}

Correspondence: John A Rumberger The Princeton Longevity Center, Princeton Forrestal Village, 136 Main Street, Princeton, NJ 08540, USA Email jrumberger@theplc.net

\begin{abstract}
The presence of mural calcification has, for decades, been recognized as a marker for atheromatous plaque in the coronary arteries and the aorta, but only in the past decade has the application of noncontrast computed tomography (CT) been shown to be a reproducible, safe, and convenient test, which now is available worldwide. However, awareness of coronary artery calcium scanning is insufficient and the practitioner must be aware of the available literature as well as understanding clinical recommendations for applications and interpretation. It is best applied in the medium/intermediate risk, asymptomatic adult regardless of ethnicity across broad age ranges for both men and women; additional prognostic information is also afforded from the calcium distribution in the coronary artery system. Additionally, information can also be derived from the same CT scan regarding heart and aorta size and assessment of the epicardial fat pad (an anatomic marker for the metabolic syndrome). Details of how this test can aid in cardiovascular risk assessment and management in adults are provided.
\end{abstract}

Keywords: coronary artery calcium, coronary artery disease, electron beam computed tomography, multidetector computed tomography, National Cholesterol Education Program Adult Treatment Plan III (NCEP ATP III), epicardial fat

\section{Introduction}

In 2004, 17.1 million individuals died of cardiovascular diseases, representing $29 \%$ of all global deaths; in the United State alone 650,000 individuals each year present with an acute coronary event as the initial symptom of developed coronary artery disease (CAD). ${ }^{1}$ In up to $20 \%$ of such individuals, this is their first and last (ie, fatal) symptom. Furthermore, it has been shown that $68 \%$ of acute coronary events ${ }^{2}$ occur in arteries that prior to the event were without a hemodynamically significant obstructive lesion as might have been defined by stress testing or direct, invasive angiography. It has been estimated that up to $75 \%$ of asymptomatic individuals destined to suffer from coronary heart disease were not aware of their sub-clinical atherosclerosis as they had no symptoms and may well have passed a standard stress test. These observations and others decry the need for a diagnostic tool to estimate focal severity of coronary sub-clinical atherosclerosis in asymptomatic individuals that is more sensitive than stress testing and a more accurate predictor of medium and long term events than office based conventional Framingham risk factor analysis.

Coronary artery calcification (CAC) and coronary atherosclerosis was described by Virchow $^{3}$ in 1858 as 'plates of bone'. In 1961 Blankenhorn $^{4}$ found, in 3,500 coronary submit your manuscript $\mid$ www.dovepress.com

Dovepress

7457 
segments from autopsies, that all CAC was associated with intimal atherosclerosis. The first description of CAC using X-ray computed tomography (CT [electron beam CT or EBT]) was in $1987 .{ }^{5}$ In 1990, Agatston and colleagues ${ }^{6}$ used high-resolution, thin collimation, 'step and shoot', ECGgated 'heartscans' and introduced the 'Agatston' total calcium scoring method, now almost universally applied in published research studies using conventional noncontrast $\mathrm{CT}$ (EBT and/or 64+-slice multidetector CT [MDCT]).

During the past 20 years, literally thousands of papers on CAC scoring have been published defining the role of the coronary calcium score in qualifying coronary atherosclerosis. In particular, data have shown its clinical value, incremental and complementary to conventional risk factors, as an aid to diagnostics and prognostication in patients at medium/ intermediate risk for coronary disease. Additionally, more recent investigations have indicated that there remains further information that can be derived from the low radiation dose, noncontrast 'heartscan'. These include data regarding the applicability of CAC across ethnic sub-groups, use of CAC for prognostication in the elderly, use of CAC in defining 'heart age', defining CAC distribution in the coronary system as an additional factor above CAC score alone, and looking beyond the coronary arteries - regarding left ventricular size, aortic root/thoracic aorta diameter, and epicardial fat.

In this context, CAC quantitation has been the subject of extensive investigations (Table 1) that have confirmed its incremental value above conventional measures of predicting

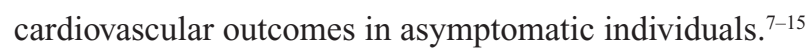

CAC scanning was incorporated into the European Guidelines on Cardiovascular Disease Prevention in Clinical Practice (2003) ${ }^{16}$ stating that "Coronary calcium scanning is thus especially suited for patients at medium risk"; thus supporting use of CAC to supplement conventional risk analysis. The American Heart Association Guidelines for Cardiovascular Disease Prevention in Women (2004) listed the finding of CAC as an example of subclinical cardiovascular disease placing certain women in the higher 10 -year risk category stating that "some patients with subclinical CVD will have >20\% 10-year CHD risk and should be elevated to the high-risk category". ${ }^{17}$

It is essential that clinicians understand what the CAC score provides in terms of diagnosis and the implications especially for medium to long term prognosis in individual patients The purpose of this review is to define a framework based upon published literature and the author's experience for the incorporation of CAC scanning into the current practice paradigm of the FRS ${ }^{18}$ and the National Cholesterol Education Program Adult Treatment Plan III (NCEP ATP III) guidelines, ${ }^{19}$ by providing recommendations for patient selection and cardiovascular risk assessment and management of medium/intermediate risk asymptomatic adults. Furthermore, new data 'beyond the coronary arteries' that can be derived from the noncontrast $\mathrm{CT}$ regarding cardiovascular health are reviewed.

\section{Overview of CAC}

EBT utilizes a rotating electron beam to acquire triggered, tomographic 100 millisecond X-ray images at $3 \mathrm{~mm}$ intervals in the space of a short breath-hold, and quantifies the calcified plaque in the epicardial coronary arteries. Current state of the art MDCT employs a rotating gantry with a special X-ray tube and 64 (or more) rows of detectors, with 165 millisecond or faster imaging at $3.0 \mathrm{~mm}$ intervals. Cardiac scans using $<64$ slice MDCT remain suspect as to their accuracy to quantify $\mathrm{CAC}$ due to motion and scan timing issues.

$\mathrm{CAC}$ is virtually always associated with mural atheromatous plaque..$^{20,21} \mathrm{~A}$ strong direct relationship has been

Table I Published prognostic studies using CT and CAC in asymptomatic individuals

\begin{tabular}{|c|c|c|c|c|c|c|}
\hline Author & $\begin{array}{l}\text { No of } \\
\text { subjects }\end{array}$ & $\begin{array}{l}\text { Mean } \\
\text { age } \\
\text { (years) }\end{array}$ & $\begin{array}{l}\text { Follow } \\
\text { up } \\
\text { duration } \\
\text { (years) }\end{array}$ & $\begin{array}{l}\text { CAC score } \\
\text { cutpoint }\end{array}$ & $\begin{array}{l}\text { Comparison } \\
\text { group }\end{array}$ & $\begin{array}{l}\text { Risk } \\
\text { ratio }\end{array}$ \\
\hline$\overline{\text { Raggi }^{7}}$ & 632 & 52 & 2.7 & Highest quartile & Lowest quartile & 13 \\
\hline Wong ${ }^{8}$ & 926 & 54 & 3.3 & Highest quartile & Lowest quartile & 8.8 \\
\hline Arad ${ }^{9}$ & 1,173 & 53 & 3.6 & CAC Score $\geq 160$ & CAC Score $<160$ & 20.2 \\
\hline Kondos $^{10}$ & 5,635 & 51 & 3.1 & CAC Score $>0$ & CAC Score $=0$ & 10.5 \\
\hline Shaw" & 10,377 & 53 & 5 & $C A C>400$ & $C A C \leq 10$ & 8.4 \\
\hline Greenland $d^{12}$ & 66 & 66 & 7 & $C A C>300$ & $\mathrm{CAC}=0 *$ & $3.9 *$ \\
\hline $\operatorname{Arad}^{13}$ & 5,585 & 49 & 4.3 & $C A C \geq 100$ & $\mathrm{CAC}<100$ & 10.7 \\
\hline Budoff' ${ }^{14}$ & 25,253 & 56 & 6.8 & $C A C>400$ & $\mathrm{CAC}=0$ & 9.2 \\
\hline
\end{tabular}

Note: *see caveat on interpretation on this study in text.

Abbreviations: CT, computed tomography; CAC, coronary artery calcification. 
established between CAC as measured by CT and both histological ${ }^{22}$ and in-vivo intravascular ultrasound ${ }^{23,24}$ measures of combined calcified and noncalcified plaque. Thus, CAC provides a viable estimate of total coronary plaque burden for a given individual and has been found to be a powerful predictor of future cardiac events, providing independent and incremental information over risk factor based assessment in the asymptomatic patient.

The original coronary calcium score as published by Agatston and Janowitz ${ }^{6}$ is determined by site-by-site calcified plaque area and calcium lesion peak intensity (density). Proper application of the 'Agatston' calcium score requires 'rules' for scanner settings (see Table 2) and any deviation from these rules invalidates the measurement. It is important to note that the original application was defined using EBT and it is essential that MDCT scanners be standardized to these parameters for any confident comparison to established scoring guidelines and for application of scoring based upon prior published works. Scanning requires a $3 \mathrm{~mm}$ CT slice thickness and a threshold for CAC of $\geq 130$ Hounsfield units (CT density) involving $\geq 1 \mathrm{~mm}^{2}$ area/lesion. MDCT scanners set to $<3 \mathrm{~mm}$ slice thickness result in 'oversampling' and calculated scores higher than that from EBT and scanners set to $>3 \mathrm{~mm}$ slice thickness result in 'undersampling' and calculated scores less than that of the EBT published standards.

Conventional categories for CAC scoring was originally put forward by Rumberger et $\mathrm{al}^{25}$ and the plaque burden quantitatively characterized as follows: the zero score (no measurable calcified plaque), a score of 1-10 as minimal, a score of 11-100 as mild, a score of 101-400 as moderate and a score $>400$ as extensive. Example images representing these categories are shown in Figure 1. The calcium volume score ${ }^{26}$ is a more reproducible parameter independent of maximum calcium density per lesion and considered to be better suited for serial studies to track progression or regression of atherosclerosis; most available computer workstations that allow convenient measurements of the calcium score report data for both the Agatston score and the volume score but most published investigations report data from the Agatston calcium score alone. By comparing a subject's Agatston calcium score to others of the same age and gender through the use of large databases of asymptomatic subjects, a calcium score percentile rank for any given individual patient can be determined. ${ }^{27,28}$ This is an index of the severity but also prematurity or, alternatively, latency of atherosclerosis development at a given chronological age and gender. Although these widely utilized nomograms are useful, it should be understood that variations according to ethnicity have been described ${ }^{29-32}$ but this subject is discussed in a later section of this manuscript.

\section{Risk stratification}

\section{Key studies}

The report of the NCEP ATP III guidelines ${ }^{33}$ made the following recommendation on the basis of existing data at the time publication (2002): "Therefore, measurement of coronary calcium is an option for advanced risk assessment in appropriately selected persons. In persons with multiple risk factors, high coronary calcium scores (eg, $>75$ th percentile for age and sex) denotes advanced coronary atherosclerosis and provides a rationale for intensified $L D L$ lowering therapy".

Subsequent to the NCEP guidelines, several major reports have highlighted the incremental value of $\mathrm{CAC}$ to conventional risk factor assessment. In a retrospective analysis Kondos et $\mathrm{al}^{10}$ (5,635 asymptomatic, predominantly low to medium conventional (Framingham) risk, middle-aged patients followed for $37 \pm 12$ months) found that the presence of any CAC by CT was associated with a relative risk (RR) for future cardiac events of 10.5 , compared to 1.98 and 1.4 for the presence of the conventional risk factors diabetes and smoking, respectively. In women, only CAC was linked to future events, with a RR of 2.6; conventional risk factors were not related. The presence of CAC also provided prognostic information incremental to the subject's chronological age.

Table 2 Cardiac CT scanning and scoring parameters for application of Agatston coronary calcium scoring (see text for details)

\begin{tabular}{ll}
\hline CT Scanner FOV & $26 \mathrm{~cm}$ \\
Minimal CT density for calcium & $\geq 130 \mathrm{HU}$ \\
CT scanner slice collimation & $3.0 \mathrm{~mm}$ \\
Minimal calcium area & $\mathrm{I} \mathrm{mm}^{2}(3$ pixels $)$ \\
Scoring by calcified lesion & calcium area $\left(\mathrm{mm}^{2}\right) \times 1$ for maximum HU I30-I99 \\
Total Agatston Score $=$ sum of all & calcium area $\left(\mathrm{mm}^{2}\right) \times 2$ for maximum HU $200-299$ \\
scores for all calcified lesions in all & calcium area $\left(\mathrm{mm}^{2}\right) \times 3$ for maximum HU $300-399$ \\
coronary arteries & calcium area $\left(\mathrm{mm}^{2}\right) \times 4$ for maximum HU $\geq 400$ \\
\hline
\end{tabular}

Abbreviations: $\mathrm{CT}$, computed tomography; FOV, field of view; $\mathrm{HU}$, hounsfield units. 

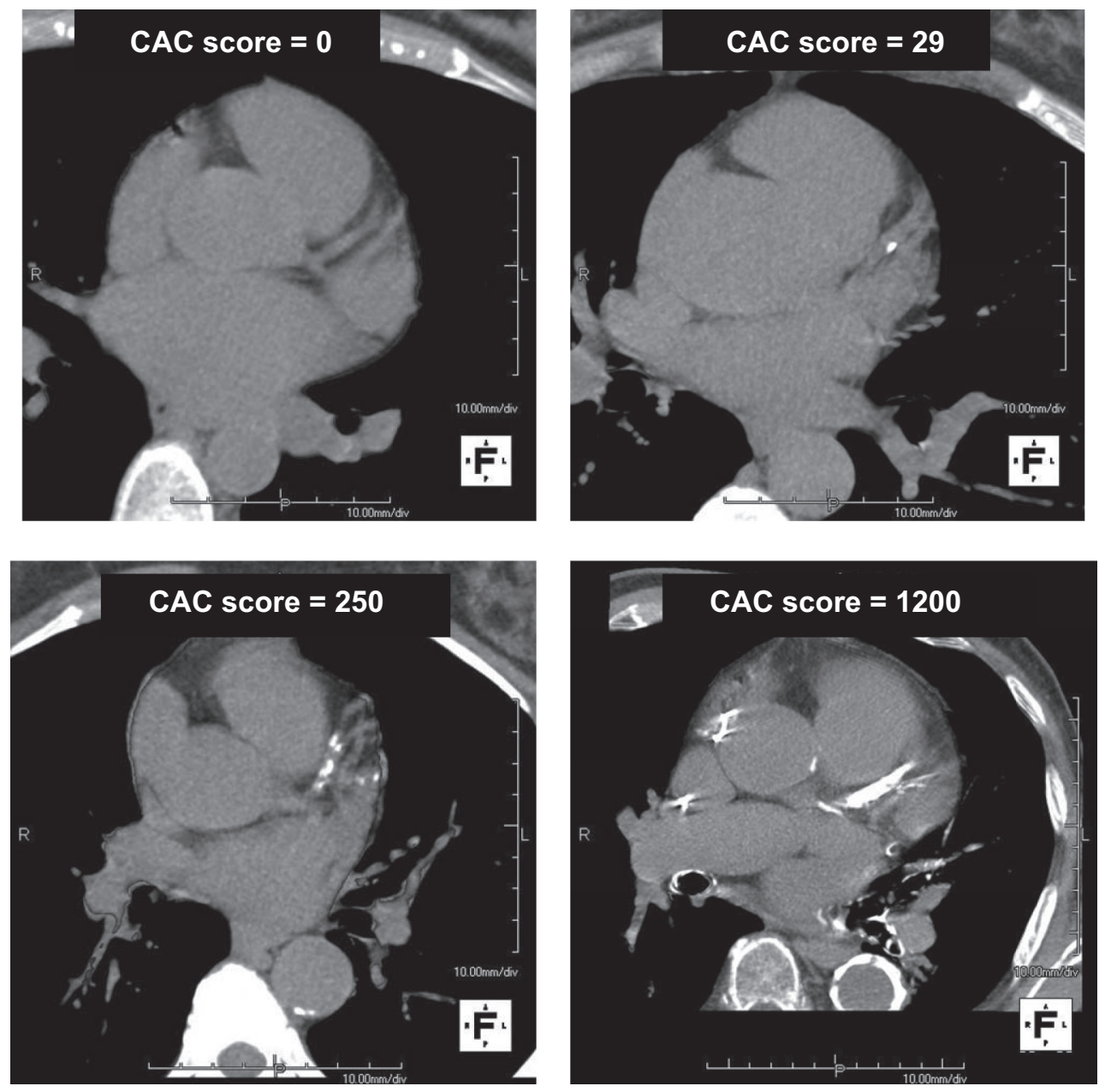

Figure I Examples of noncontrast coronary calcium CT scans at the base of the heart: top left, CAC score =0; top right, CAC score = 29; bottom left, CAC score = 250; bottom right, CAC score $=1200$.

Abbreviations: CT, computed tomography; CAC, coronary artery calcification.

Shaw et a $\mathrm{l}^{11}$ retrospectively analyzed 10,377 asymptomatic patients with a 5-year follow up after an initial noncontrast CT evaluation. All-cause mortality (United States National Death Index listing at follow up) increased proportional to baseline CAC score, which was an independent predictor of risk after adjusting for all Framingham risk factors $(P<0.001)$. Superiority of CAC to conventional Framingham risk factor assessment was also demonstrated by a significantly greater area under the receiver operating characteristic (ROC) curves ( 0.73 vs $0.67 ; P<0.001)$. Incremental value of CAC to Framingham risk was also established by a significant increase of the area under the ROC curves, from 0.72 for Framingham risk to 0.78 with the addition of CAC $(P<0.001)$. Stratification of allcause mortality risk by CAC score was as effective in women as in men. A recent study published by Budoff et al using also the National Death Index looked at all-cause mortality in $>25,000$ initially asymptomatic subjects and found similar data for prognostication using the baseline or initial CAC score. ${ }^{14}$
Greenland et $\mathrm{al}^{12}$ analyzed a population based study of 1,461 prospectively followed, older asymptomatic subjects, who were predominantly medium to high risk, and found that CAC scores $>300$ significantly added prognostic information to Framingham risk analysis in the intermediate, $10 \%-20 \%$ Framingham risk category.

In the Saint Francis Heart Study, ${ }^{13}$ a prospective, population based study of 5,585 asymptomatic patients, CAC scores $>100$ were associated with RR from 10.4 to 32 , and transformed conversion of Framingham intermediate risk individuals to high or very high risk status. In a subset of 1,817 patients with clinical risk factor data, incremental information over Framingham scores was documented, with areas under the ROC curves of 0.79 for CAC and 0.69 for Framingham $(P=0.0006)$. The greatest separation of patients who developed nonfatal myocardial infarction (MI) or cardiac death in follow-up from those who did not was at the Agatston CAC score of 100; which has for nearly the 
past decade been considered the cutpoint between mild and moderate coronary atherosclerosis. ${ }^{25}$ Importantly, the $15 \%$ of patients in the Saint Frances study with initial CAC scores in the 100-400 range had a 10-fold increase in RR for any cardiovascular event compared to the $33 \%$ of individuals entered into the study protocol with a zero CAC score.

\section{Applications of total CAC score in ethnic sub-groups}

In non-Hispanic Caucasians, CT 'heartscan' derived measures of the total CAC score predict incident coronary heart disease independent of traditional coronary risk factors, as discussed above. However, it is has been controversial as to whether the CAC score predicts coronary heart disease and prognosis across racial or ethnic subgroups. The Multi-Ethnic Study of Atherosclerosis (MESA) collected data on risk factors and performed noncontrast $\mathrm{CT}$ for coronary calcium in a population-based, multiethnic sample of 6,722 men and women, of whom 38.6\% were Caucasian-American, 27.6\% were African-American, 21.9\% were Hispanic-American, and 11.9\% were Chinese-American. The subjects had no known clinical cardiovascular disease at study entry and were followed for a median of 3.8 years. In comparison with subjects with no coronary calcium (ie, a CAC score of zero), the adjusted risk of a coronary event was increased by a factor of 7.73 among participants with coronary calcium scores between 101 and 300 and by a factor of 9.67 among participants with scores above 300 ( $P<0.001$ for both comparisons). ${ }^{34}$ Among the four ethnic sub-groups, a doubling of the calcium score increased the RR of a major coronary event by $15 \%$ to $35 \%$ and the risk of any coronary event by $18 \%$ to $39 \%$. Furthermore, the areas under the ROC curves for the prediction of both major coronary events and any coronary event were higher when the calcium score was added to standard risk factors. The investigators concluded that the total Agatston CAC score was a strong predictor of incident coronary heart disease and provided similar predictive information beyond that provided by standard risk factors in each of the four major racial/ethnic sub-groups investigated.

\section{Application of total CAC scores in the elderly}

The majority of data using the predictive power of the total Agatston CAC score have been derived from studies done in individuals between 40 and 65 years of age. It is well known that the CAC score increases as a function of age in both men and women and many have suggested that the predictive power of the CAC score may be diminished in the elderly in whom the prevalence of any CAC is common.

Raggi et $\mathrm{al}^{35}$ reported on the predictive power of the total CAC score in a total of 35,383 individuals referred by their primary care physician for a 'heartscan'. Of this group, 3,570 were above the age of 70 years. The investigators evaluated the predictive power of CAC scoring in 6 age deciles from individuals $<40$ to $>80$ years of age. The study reaffirmed that overall mortality rate increased with each age decile (hazard ratio $[\mathrm{HR}], 1.09$ ) with mortality rates greater for men than women (HR, 1.53; $P<0.0001)$. The authors concluded that $\mathrm{CAC}$ risk was different in men and women but that even in elderly patients, CAC score was an independent prognostic indicator.

\section{Defining heart age vs chronological age}

The Framingham risk score (FRS) assesses future 'risk' from standard risk variables including chronological age of the patient, gender, total cholesterol value, high-density lipoprotein (HDL) cholesterol value, smoking history, and blood pressure (noting if the patient is or is not currently taking hypertensive medications). Of these values, the variables with the greatest influence within the FRS model are age and gender. Overall, the FRS defines the 'median' risk for a group of individuals of similar age and risk factor status, however conceptually this can be further individualized through refinement of patient age as 'heart age' for the FRS calculation. Grundy ${ }^{36}$ was the first to suggest this approach. Nassir and Rumberger ${ }^{37}$ expanded on this concept by redefining the traditional FRS scoring tables substituting 'heart' age for chronological age using percentile ranking of total CAC scores.

Estimation of an individual's 'heart age' can be done in a straightforward manner using previously published database information. Figure 2 shows a graphical representation of CAC score data from asymptomatic men and women originally presented by Hoff et al. ${ }^{27}$ Upon this figure is indicated information from a male aged 50 years with a total CAC score of 100 and a female, aged 55 years, with a total CAC score of 50. The 'median' CAC score for a 50-year-old man is 15 and the corresponding 'median' score for a 50-year-old woman is 1. From the data as shown in Figure 2, these individual total CAC scores are more representative of median scores from a man age 60 years (ie, 10 years older) and a woman age 70 years (ie, 15 years older). To illustrate further, assume that both are nonsmokers, have a total cholesterol value of $5.83 \mathrm{mmol} / \mathrm{L}$ (225 mg/dL), an HDL of $1.09 \mathrm{mmol} / \mathrm{L}$ (42 mg/dL), and both have mild, untreated hypertension with resting systolic 


\section{Total CAC percentiles by age}

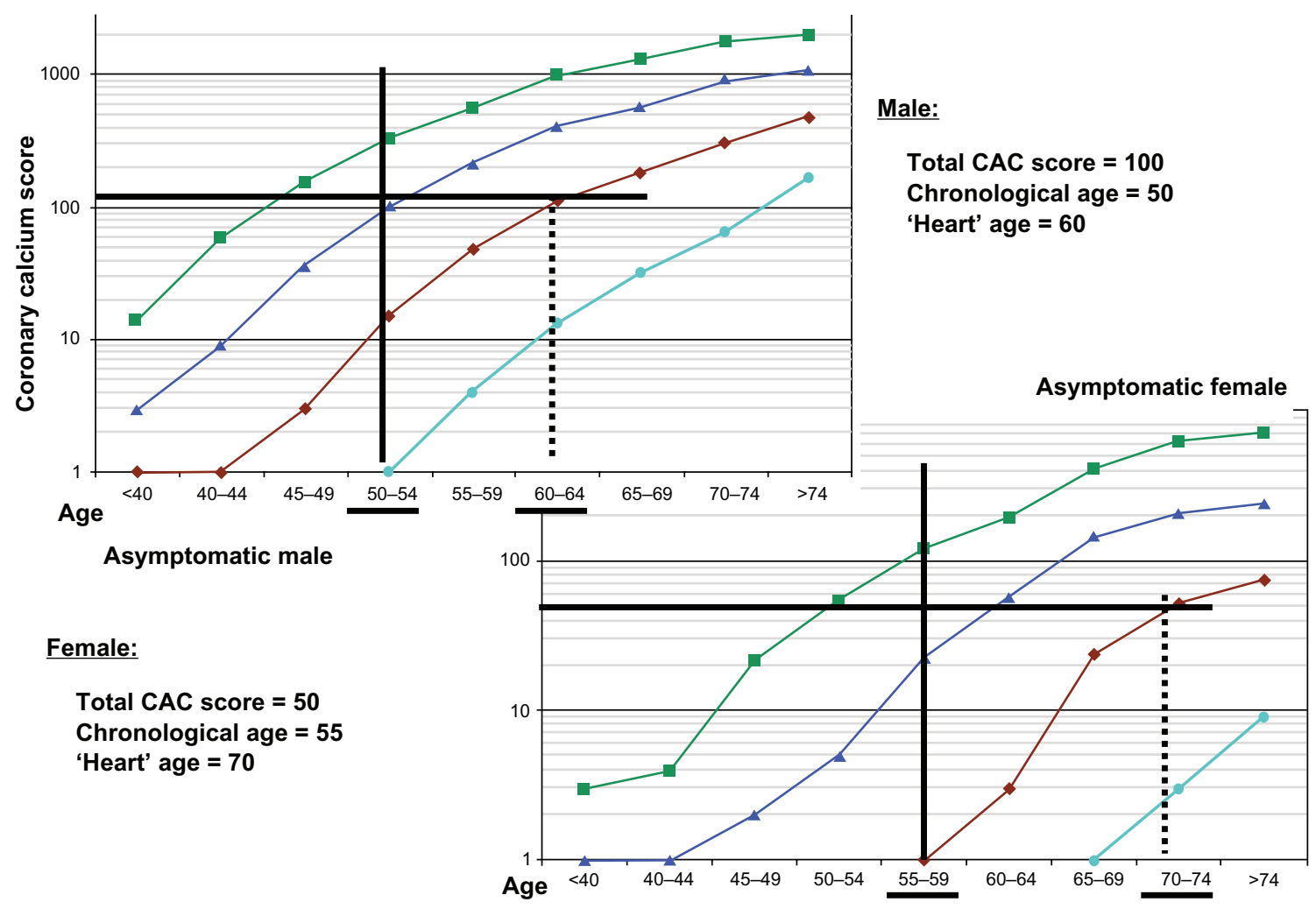

Figure 2 Graphical representation of data presented by Hoff ${ }^{27}$ showing distribution of total CAC scores in 35,246 men and women. The red line represents the median score as a function of age. Superimposed are information regarding determination of 'heart age' (see text for details) in a 50 year old man with a CAC score of 100 and a 55 year old woman with a CAC score of 50 .

Abbreviation: CAC, coronary artery calcification.

blood pressures of $135 \mathrm{mmHg}$. The subsequent calculated Framingham risk $^{18}$ for the man (based on chronological age) would be $0.7 \% /$ year and for the woman $0.2 \% /$ year. Using the 'heart age' (but not changing the point scores based on chronological age points accumulated for the other risk factors) the cardiovascular 'risk' for developing symptomatic disease would be $1.2 \%$ /year and $0.6 \%$ year for the man and women, respectively. These revised risk estimates can then be used clinically to guide the level of aggressiveness for medical interventions. Heart age and a FRS integrating conventional risk factors and a given individual's CAC score can be conveniently calculated at the MESA website at http:// www.mesa-nhlbi.org/CACReference.aspx.

\section{Coronary calcium pattern and distribution}

The majority of data regarding the prognostic power of CAC has been reported using the total Agatston coronary calcium score whereas little has been published regarding calcification patterns and prognosis. A common question is: "does a total calcium score of $X$, attributed to a single site in a single coronary artery invoke the same prognostic power as the same total calcium score in an individual where the distribution is diffuse and found in three separate coronary arteries or the left main artery?"

Ehara et $\mathrm{al}^{38}$ studied preintervention intracoronary ultrasound in patients with acute coronary syndromes and in patients with stable coronary artery disease (CAD) and noted that 'spotty' coronary calcium (ie, a focus $<3 \mathrm{~mm}$ in size) was more likely to be associated with an acute presentation. Schmermund et al had noted previously that a pattern of 'spotty' (mild to moderate diffuse versus moderate to severe focal) coronary calcium by $\mathrm{CT}$ was more commonly associated with diffuse coronary plaque and focal outward (ie, positive) coronary artery remodeling at angiography. ${ }^{39}$ Most recently, Williams et $\mathrm{a}^{40}$ reported on 14,759 individuals for all-cause mortality at an average of 6.8 years of follow up. They noted that the mortality rate exceeded $2 \%$ per year (considered 'coronary artery disease' risk equivalent by the NCEP) in the setting of more than 20 calcified lesions. Additionally, the finding of CAC in the left main coronary artery as opposed to any other anatomic site was associated with increased all-cause mortality. 
Quantification of the distribution of coronary artery calcium was recently proposed with a 'calcium coverage score' (CCS) calculated as the proportion of $5 \mathrm{~mm}$ coronary arterial segments affected by calcific plaque..$^{41}$ In the MESA, after 41 months of follow-up, a two-fold increase in CCS was associated with a $34 \%$ increased risk of a hard coronary disease event, defined as definite adjudicated myocardial infarction (MI), coronary death, and/or angina, compared with just a $14 \%$ increased risk indicated by the total CAC Agatston score. Additional validation work is needed on the CCS, but in the future, application of the CCS may result in further refinement in the assessment of individual risk over and above the total CAC score.

\section{The zero score}

Asymptomatic individuals with zero CAC score have not yet developed detectable, calcified coronary plaque but may have fatty streaking and early stages of a plaque and have a $1 \%-2 \%$ chance of focal obstructive coronary artery disease. Atherosclerotic plaques are present in many young adults, ${ }^{42}$ but the event rate in patients with CAC score of zero is very low. ${ }^{7,10,12,13}$ Raggi et $\mathrm{al}^{7}$ have demonstrated an annual event rate of $0.11 \%$ in asymptomatic subjects with 0 scores, and in the St Francis Heart Study, ${ }_{13}^{13}$ scores of 0 were associated with a $0.12 \%$ annual event rate over the ensuing 4.3 years. Greenland et al ${ }^{12}$ in a higher risk asymptomatic cohort, noted a higher annual event rate $(0.62 \%)$ with $0 \mathrm{CAC}$ scores; however a less sensitive CAC detection technique (significant 'under sampling' and thus 'under-scoring' by using higher thresholds for positive scans, ie, nonstandard Agatston scoring) and marked ethnic heterogeneity may have contributed to their different findings. ${ }^{43}$

A more definitive study (again using the National Death Index) which addressed the issue of a zero CAC score and all-cause mortality was reported by Blaha and colleagues. ${ }^{44}$ Annualized all-cause mortality was assessed in 44,052 consecutive asymptomatic adults (age $54 \pm 10$ years) referred for risk assessment using noncontrast $\mathrm{CT}$ and $\mathrm{CAC}$ scoring. Follow up was done at $5.6 \pm 2.6$ years. A total of 19,898 subjects had no detectable CAC on initial cardiac $\mathrm{CT}$ examination. Annualized all-cause mortality rates for $\mathrm{CAC}=0$ were 0.87 deaths $/ 1,000$ person years $(0.087 \% /$ year). Mortality rates for a CAC score of $1-10$ and $>10$ were higher at 1.92 and 7.48/1,000 person years, respectively. This confirms that a zero CAC score in an asymptomatic individual puts them at a $<0.1 \%$ year mortality risk, but scores above zero represent incrementally and substantially increased cardiac risk.

\section{Distribution of CAC}

There is often confusion as to what would be the typical distribution of calcium scores as applied to an asymptomatic group of intermediate risk individuals. Misconceptions abound as many symptomatic individuals undergoing CT coronary angiography are often found to have very high calcium scores and many physicians that I have consulted with seem to think that 'everybody who is middle aged has some $C A C^{\prime}$. This is simply not true.

As an example, I offer information on 5,192 randomly assessed, asymptomatic individuals referred to me personally for cardiovascular risk assessment by their primary care doctors. The average age was $53 \pm 10$ years $(60 \%$ men); $31 \%$ had total cholesterol above $5.18 \mathrm{mmol} / \mathrm{L}(200 \mathrm{mg} / \mathrm{dl})$, $22 \%$ had a systolic blood pressure $>140 \mathrm{mmHg}, 7 \%$ were current smokers, and $23 \%$ had a family history of premature heart disease in a first degree relative. Calcium scores ranged from 0 to 5808 (mean $128 \pm 378$ ). A zero CAC score was found in $47 \%$ while a score above 1000 was found in only $3.5 \%$. A pie chart of the distributions of calcium scores in this asymptomatic group is found in Figure 3.

\section{Clinical applications \\ Patient selection}

Recommendations for CAC scanning are not based on age and gender alone. Rather, the FRS, which incorporates both age and gender, is recommended as the initial step in selecting the appropriate test populations. Asymptomatic patients in the $10 \%-20 \%$ Framingham 10 year risk category (medium/ intermediate risk) comprise of the group that presents the greatest challenge to the treating physician, and are those in whom the application of CAC scoring is considered most appropriate. This group represents up to $40 \%$ of the population that might be seen sitting in the waiting room of the average internist. ${ }^{45}$ While this application was proposed as being reasonable in the early American College of Cardiology/American Heart Association (ACC/AHA) consensus statement, ${ }^{46}$ the recent additional evidence of risk-stratification by $\mathrm{CAC}$ in this group has resulted in a greater acceptance of its benefits, and was included in the 2005 AHA Scientific Statement on noninvasive testing in women for use in the intermediate risk population. ${ }^{17}$

Selected patients with less than intermediate Framingham risk may also benefit. For instance, most young patients with a family history for premature $\mathrm{CAD}$ (1st degree relatives with documented heart disease below the age of 55) will not have sufficient risk factors to even warrant Framingham scoring (lower NCEP risk), or will be in the low (1\%-10\%) 10 year Framingham risk group. ${ }^{18,47}$ Data from Schmermund et $\mathrm{a}^{24}$ and 

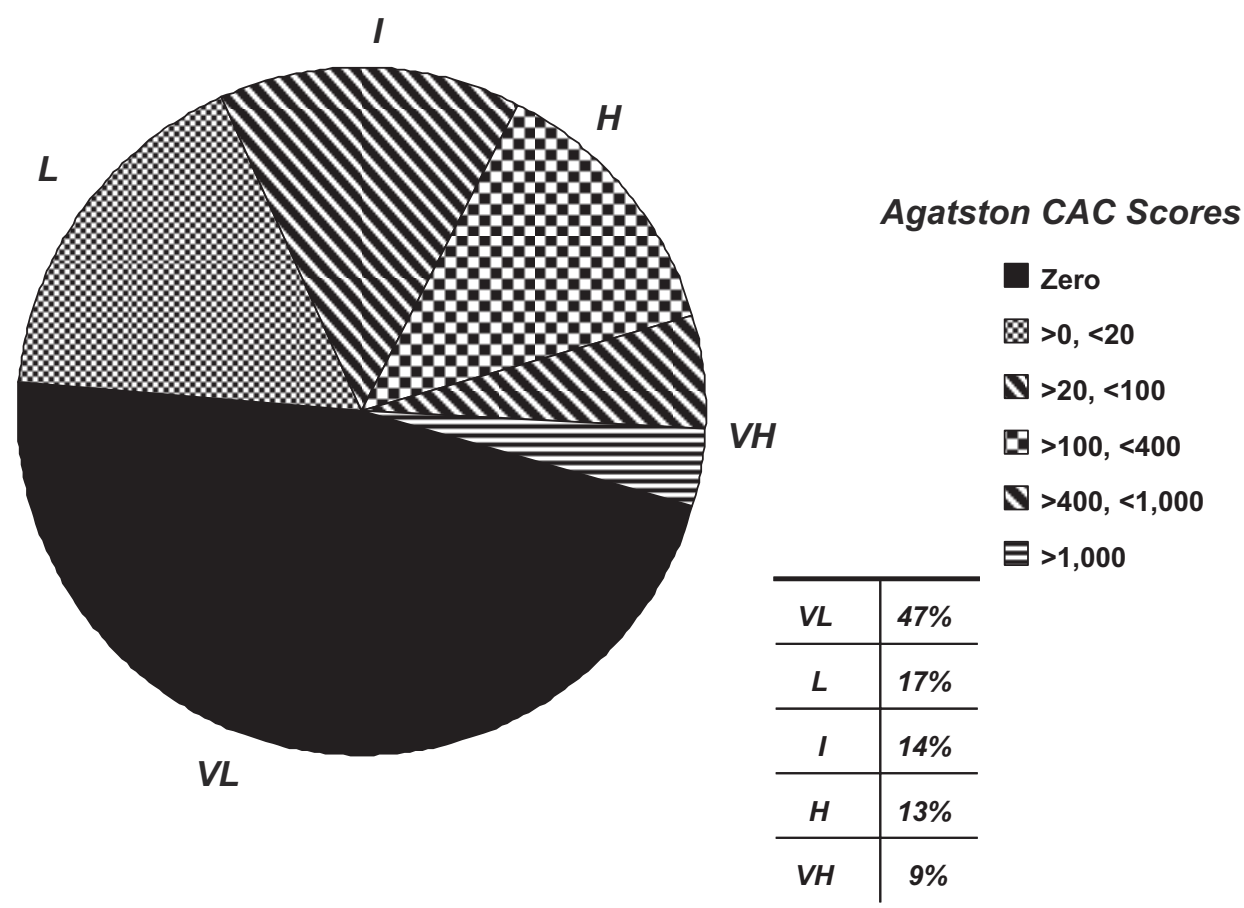

Figure 3 Pie chart representing the distribution of coronary calcium scores in >5,000 asymptomatic, middle aged adults referred to one scanning center for assistance in cardiovascular risk stratification. Subsequent stratification of risk is altered based upon the total calcium scores (see text for detail) as very low (VL $=<0.1 \% / y e a r)$, low $(\mathrm{L}=<1 \% /$ year), true intermediate $(\mathrm{I}=\mathrm{I} \%-2 \% /$ year $)$, high $(\mathrm{H}=>2 \% /$ year $)$, and very high $(\mathrm{VH}=>3 \% /$ year $)$.

Pohle et a ${ }^{48}$ indicate that $95 \%$ of acute MI patients would have been identified by CAC plaque imaging, even in those with a mean age of 41 years. On the basis of these observations, the use of CAC scoring should be considered in patients with a family history of premature CAD, especially if their Framingham risk is intermediate (although many would advocate this use even if the initial Framingham risk was calculated as low; since family history remains one of the most positive risk factors and is NOT considered part of conventional Framingham Risk scoring).

Selective application of CAC scanning to patients with Framingham high risk ( $>20 \% /$ decade or $>2 \% /$ year) may also be warranted. For instance, some Framingham high risk patients may be intolerant of statins or may strongly prefer alternativemedicine approaches. In these patients, CAC confirmation of high risk may be used to reinforce the necessity for finding a statin that can be tolerated and for persuading the refractory patient of the need for aggressive treatment. Conversely, the absence of any CAC for age or gender may permit relaxation of the treatment goals, an approach that appears justified by the low event rate in the 0 score CAC group. . $^{710,12-14}$

\section{Initiation and goals of drug therapy}

The presence or absence and amount of CAC can be useful for clinical decision making, as previously recommended in the AHA Prevention V Update. ${ }^{49}$ As an extension of this report, based on recent data, Table 3 provides simple, easily implemented treatment paradigms for combining risks of varying CAC scores with the most recent NCEP recommendations. Patients in the $10 \%-20 \% 10$ year risk category that are identified to be at higher risk by CAC become candidates for secondary prevention lipid goals regardless of their baseline lipid level. This would apply even for patients with low-density lipoprotein (LDL) cholesterol $<2.59 \mathrm{mmol} / \mathrm{L}(<100 \mathrm{mg} / \mathrm{dL})$, as implied by the Heart Protection Study ${ }^{50}$ and stated in the prior NCEP report.

Based on prognostic data, CAC scores $>100$ and/ or $>75$ th percentile for age/gender serves to define a CAD risk equivalent (ie, $>20 \%$ over the next decade). In the $\mathrm{St}$ Francis Heart Study, ${ }^{13}$ the CAC cutpoint for secondary prevention risk equivalency in the Framingham intermediate $10 \%-20 \% 10$ year risk group was a score $>100$; or, additionally at a score $>75$ th percentile for age as suggested by the NCEP guidelines. In this regard, CAC scores $>400$ or $>90$ th percentile for age/gender are associated with a very high annual risk ( $4.8 \%$ and $6.5 \%$ respectively), ${ }^{7,51}$ and these individuals are candidates for an even more aggressive approach (ie, $\mathrm{LDL}<1.81 \mathrm{mmol} / \mathrm{L}(<70 \mathrm{mg} / \mathrm{dL})$ as suggested by the latest update to the $\mathrm{NCEP}^{19}$ ) and possibly further stratification with stress testing (see Discussion below). 
Table 3 Guidelines for treatment of LDL cholesterol in asymptomatic individuals classified as intermediate risk patients by NCEP (Framingham 10\%-20\% 10 year risk)

\begin{tabular}{|c|c|c|c|}
\hline $\begin{array}{l}\text { CAC score and } \\
\text { percentile ranking }\end{array}$ & $\begin{array}{l}\text { Framingham risk group } \\
\text { equivalent }\end{array}$ & $\begin{array}{l}\text { Target LDL goal } \\
\mathrm{mmol} / \mathrm{L}(\mathrm{mg} / \mathrm{dl})\end{array}$ & $\begin{array}{l}\text { Pharmacologic therapy } \\
\text { indicated @ mmol/L (mg/dl) }\end{array}$ \\
\hline Zero & Very low risk ( 10 year risk < $1 \%$ ) & $<4.14(160)$ & $\geq 4.92(190)$ \\
\hline$>0,<10$ AND & Low risk $(10$ year risk $>1 \%$ & $<3.37(130)$ & $\geq 4.14(160)$ \\
\hline$<75$ th percentile & but $<10 \%)$ & & \\
\hline $1 \mathrm{I}-100$ AND & Intermediate risk ( 10 year & $<3.37(130)$ & $\geq 3.37(130)$ \\
\hline$<75$ th percentile & risk $>10 \%$ but $<20 \%$ ) & & \\
\hline $101-400$ OR $\geq$ & High risk (coronary disease & $<2.59(100)$ & $\geq 2.59(100)$ \\
\hline 75 th but $<90$ th & risk equivalent; 10 year & & \\
\hline percentile & risk $\geq 20 \%$ ) & & \\
\hline$>400$ OR $\geq 90$ th & Very high risk ( 10 year & $<\mathrm{I} .29-\mathrm{I} .8 \mathrm{I}(50-70)$ & any LDL level \\
\hline percentile & risk $>30 \%)$ & & \\
\hline
\end{tabular}

Abbreviations: LDL, low-density lipoprotein; NCEP, National Cholesterol Education Program; CAC, coronary artery calcification.

In the Framingham 10\%-20\% 10 year risk population, patients with CAC scores $\leq 100$ and/or $\leq 75$ th percentile remain in the same risk group or are transformed to lower risk categories depending on the actual score or percentile rank. A reasonable approach is to leave the patients with $\mathrm{CAC}$ scores from $10-100$ and $<75$ th percentile in the intermediate risk (10\%-20\% 10 year risk), and reclassify patients with CAC scores from $1-10$ and $<75$ th percentile as low risk $(<10 \%$ 10 year risk) and treat accordingly. CAC scores of 0 would reclassify the patient to the very low risk category.

As noted above, some patients in the lower risk groups based on Framingham scores, such as younger patients (35-45 years of age) with a strong family history of premature coronary heart disease, may be appropriately tested with CAC scanning. In such patients, the recommendations in Table 3 would also apply. While it is widely accepted that high CAC scores reinforce the intensity of medical therapy, how low CAC scores should affect therapy is not yet clear. It would appear reasonable that in high risk asymptomatic patients who have undergone imaging, $C A C$ scores $\leq 100$, and in particular, $\leq 10$, imply a lower than expected risk and may reduce the intensity of therapy. The rationale for this lowest category is as follows. If the use of cholesterol lowering medications (such as statins) can across the board, lower the risk of an MI by $1 / 3$, this is significantly of value when the risk is found to be high, but is less practical when the risk is very low; for instance, if the 'risk' of a cardiovascular event is $0.1 \%$ /year for a zero CAC score (as supported by the published literature), then using a cholesterol medication may be of limited incremental value if the risk reduces to $0.07 \%$.

It is important to note, however, that a CAC score of 0 does not imply that no treatment is necessary. Rather, it is used to shift the patient to a lowest risk group. For example, early information has shown that the event rate in diabetics with $0 \mathrm{CAC}$ scores is as low as in nondiabetics with 0 scores, ${ }^{52}$ potentially creating a group of diabetics who would not have to be considered to have 'coronary heart disease' equivalence. More data is needed in these groups for determining the therapeutic implications of the absence of CAC.

\section{Evaluation of treatment}

The use of changes in CAC score rather than changes in lipid values to track treatment effects has been under investigation. Serial CT scanning has shown that aggressive lipid lowering therapy may slow progression of calcified plaque ${ }^{53-58}$ although it is far from infallible. ${ }^{59}$ Clearly, a noninvasive tool with which sequential testing could be performed safely and reliably would be highly desirable provided the results are associated with significant prognostic value. Raggi et al have demonstrated that CAC progression was greater in patients with future $\mathrm{MI}^{58}$ whereas LDL levels on treatment were similar in patients with and without events. Progression was associated with a worse prognosis compared to stabilization, irrespective of baseline CAC score. However, more studies are required to justify the broad based use of serial CAC scanning to monitor treatment efficacy.

\section{Triage for stress testing}

The asymptomatic middle aged population has a low pretest likelihood for significant obstructive disease ( $>50 \%$ stenosis); nonetheless, stress testing in this group is common even though there are no guideline sanctioned recommendations. Bayes Theorem ${ }^{60}$ dictates that stress testing of any kind will yield a large proportion of false positives in this low pretest likelihood group. CAC scoring is better suited than stress testing as the initial assessment; stress testing may be of value in selected patients after the CAC test to determine whether ischemia is present in order to further guide management 
and in particular to determine whether coronary angiography should be considered. Stress testing performed to evaluate cardio-respiratory fitness and blood pressure response to exercise should not be influenced by CAC.

Based on studies in relatively large groups of patients, it is clear that stress testing is not needed in the vast majority of asymptomatic patients with CAC scores $>0$ (see score distributions in a sample of such patients in Figure 3). In 411 patients without established coronary disease undergoing CAC scanning and myocardial perfusion imaging (MPI), He et $\mathrm{al}^{61}$ found $0 \%$ positive nuclear tests with CAC scores of 0 and 1-10, 2.6\% with CAC scores 11-100, 11.6\% with CAC scores $101-399$, and $46 \%$ with CAC scores $\geq 400$. However, these high percentages of abnormal MPI in various CAC score categories are at odds with the observations of Berman et al. ${ }^{62}$ In 1195 patients without known disease, Berman noted 1.6\% abnormal MPI with CAC scores of $0,0 \%$ with scores of $1-9$, $2.4 \%$ with scores of 10-99, 5.2\% with scores of 100-399, $8.9 \%$ from $400-999$, and $19.9 \%$ with scores $\geq 1000$. However, both studies concluded that sufficient pretest likelihood of an abnormal MPI is present in the CAC score $>400$ group and in only selected patients in the 100-400 CAC score group. Many clinicians now suggest stress testing when the baseline CAC score exceeds 400 , even if they are asymptomatic. This was the same conclusion reached by Rumberger and colleagues in the first guidelines paper on CAC scoring, published more than a decade ago. ${ }^{25}$

The study of Berman et al also illustrated that the normal MPI study alone, performed without the CAC score, was insufficient to place patients into a group not requiring aggressive medical therapy, even if the short term prognosis is benign. They found that $56 \%$ of 1195 patients with normal MPI had CAC scores $>100$, and $31 \%$ had CAC scores $>400$. Thus, if the likelihood of CAD was sufficiently high to warrant stress testing with any modality, CAC scanning should be considered after a normal stress evaluation to guide the aggressiveness of medical therapy. ${ }^{62}$

Regardless of the CAC score, stress testing should precede coronary arteriography in the asymptomatic patient, and coronary angiography should be reserved for those patients deemed to be at high risk on the basis of the stress test results. However, this group may alternatively be considered for direct CT angiography (CTA) using 64+-slice MDCT.

\section{Limitations}

As with any diagnostic method, CAC testing has limitations. It does not evaluate the degree of coronary stenosis; the specificity of a positive CAC score for the presence of obstructive disease is in the $40 \%$ range. ${ }^{63}$ In addition, the specificity of CAC for cardiac events is quite low, since only a minority of patients with CAC experience events. However, the specificity of CAC for the diagnostic goal, the identification of sub-clinical atherosclerosis, is nearly $100 \%$. CAC does not visualize noncalcified (incorrectly deemed 'soft') plaque, and patients with exclusively noncalcified plaque will escape detection; however, although discussed by clinicians, such a situation of significant noncalcified plaque in the absence of concomitant calcified plaque somewhere within the coronary system is rare in the pathology literature.

CAC scanning is associated with a radiation dose which ranges from $0.9 \mathrm{mSv}$ (milli-Severt) for EBT and up to $2.5 \mathrm{mSv}$ for prospectively gated MDCT. However, awareness of the need to reduce radiation doses for routine $\mathrm{CT}$ scanning has resulted in major improvements in scanning algorithms from the MDCT manufacturers. Currently, a retrospective CAC scan can be done in almost all situations with radiation doses $<1.5 \mathrm{mSv}$ and commonly at $<1 \mathrm{mSv}$ with the latest scanners (the radiation dose for a standard two-view mammogram is about $0.75 \mathrm{mSv}$, to put this in perspective). However, the fact that a cardiac CT examination of any type does necessitate radiation exposure to the patient emphasizes the importance of appropriate patient selection to ensure that the benefits outweigh the risks.

The major limitation of this report is the absence of evidence based confirmation of the CAC based proposed modifications of the NCEP-III guidelines, and the reliance on the clinical experience of the authors and extrapolation of risk from key clinical studies, some of which are retrospective.

\section{Beyond the coronary arteries}

A noncontrast $\mathrm{CT}$ of the heart can provide additional anatomic information beyond just defining the amount or pattern of $\mathrm{CAC}$. There has been renewed interest in using all potential scan information as it might relate to an individual's development of or risk for developing cardiac disease. In particular three new areas have been most recently examined.

\section{Heart size}

Nasir et $\mathrm{al}^{64}$ used data from the MESA study to compare left ventricular size from noncontrast $\mathrm{CT}$ scans of the heart with magnetic resonance imaging (MRI) determinations of left ventricular muscle mass and volumes in the same subjects. A total of 5,004 participants were included in the investigation. From the 'heartscan', a single midventricular CT 

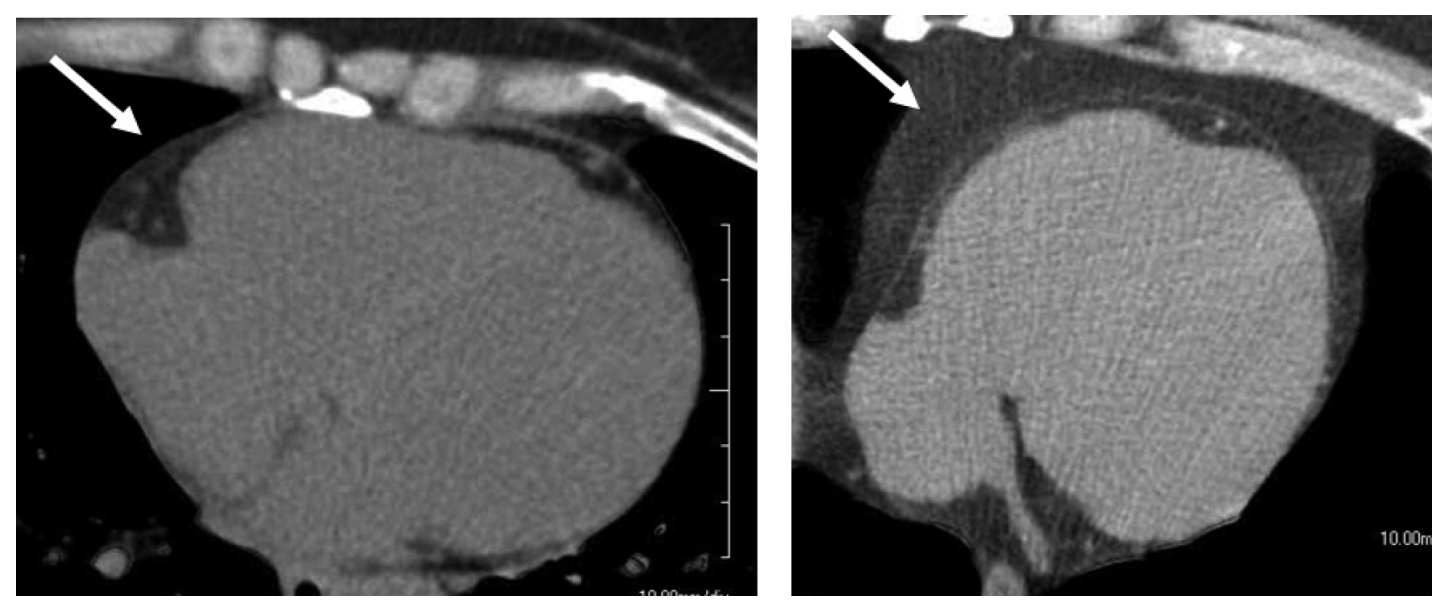

Figure 4 A single, mid LV slice from a noncontrast CT of the heart (ie, a 'heartscan') demonstrating the epicardial fat distribution in a patient without metabolic syndrome (Left arrow) and a patient with known metabolic syndrome (right arrow). See text for details.

Abbreviations: LV, left ventricle; CT, computed tomography.

slice was defined at the level containing the coronary sinus or the first level below the left atrium. Then a straight line connecting the anterior-posterior junction of both ventricles was drawn to divide the left and right ventricles and the subsequent area of the left ventricle was then measured with planimetry and left ventricular (LV, middiastolic) volume was estimated using a previously validated algorithm developed by Mao. ${ }^{65}$ Left ventricular measurements with $\mathrm{CT}$ correlated well with MRI regarding both $\mathrm{LV}$ volume $(\mathrm{r}=0.73 ; P<0.01)$ and LV muscle mass $(\mathrm{r}=0.74 ; P=0.01)$. Further studies will be required, but these data clearly indicate that an additional important parameter for cardiovascular diagnosis and prognosis, namely estimates of LV volume/mass, are possible from a standardized 'heartscan'.

\section{Thoracic aorta size}

Dilation of the aortic root and the thoracic aorta is common in aortic valve disease, primary diseases of the aorta, and systemic hypertension. Two-dimensional echocardiography is most commonly used clinically for these measurements; however, similar anatomic areas are also imaged as part of a standard 'heartscan'. Lin et al ${ }^{66}$ evaluated noncontrast, ungated, scans of the chest/heart in 103 normotensive, nonobese individuals who were free of known cardiovascular disease. They reported that normal aortic root size $(2.5-3.7 \mathrm{~cm})$, ascending aorta diameter $(2.1-3.5 \mathrm{~cm})$, and thoracic aorta $(1.7-2.6 \mathrm{~cm})$ diameters were very similar to what had been previously reported using standard transthoracic echocardiography. These data imply that prospective application of these normative values to individuals undergoing 'heartscans' for assessment of coronary calcium may provide additional diagnostic information.

\section{Pericardial/epicardial fat}

Visceral abdominal/adipose fat has a known association with the metabolic syndrome ${ }^{67}$ and its measurement from abdominal CT scans has been standardized for some time. ${ }^{68}$ Recently, an association of visceral adipose fat with epicardial adipose fat on MDCT scans has been noted ${ }^{69}$ where both were found to correlate with body mass index, waist circumference, and other known cardiovascular risk factors such as coronary and vascular calcification. Recently Nichols and colleagues ${ }^{70}$ have evaluated a three-dimensional volumetric measure of epicardial fat using contrast-enhanced MDCT and have found it be highly reproducible. However, further data are needed to determine the algorithms application to the more commonly obtained noncontrast scans done as part of standard CAC assessment. An example of a single slice, mid LV 'heartscan' from a patient without and a patient with metabolic syndrome, demonstrating the epicardial fat distribution, is shown on Figure 4. A reliable and reproducible measure of epicardial fat would provide yet another potential for the 'heartscan' to be used in cardiovascular risk stratification, especially in individuals with the metabolic syndrome.

\section{Conclusions}

The increasing use of CAC scanning for risk assessment is now supported by extensive evidence in appropriately selected patients. Critical to its implementation is the ability of practitioners to understand the CAC scoring data and limitations of the test, as outlined in this review. Only then can they appropriately utilize this knowledge, including data 'beyond the coronary arteries', to improve cardiovascular risk assessment and management in their individual patients. 


\section{Disclosure}

The author reports no conflicts of interest in this work.

\section{References}

1. Heart and Stroke Statistical Update. 2001. Dallas, TX: American Heart Association.

2. Falk E, Shah PK, Fuster V. Coronary plaque disruption. Circulation. 1995;92:657-671.

3. Virchow R. Die Cellulärpathologie in Ihrer Begrundung auf physiologische und pathologische Gewebesslehre. Berlin, August Hirschwald, 1858.

4. Blankenhorn DH. Coronary arterial calcification: a review. Am J Med Sci. 1961;242:1-9.

5. Tannenbaum SR, Kondos GT, Veselik KE, Prendergast MR, Brundage BH, Chomka EV. Detection of calcific deposits in coronary arteries by ultrafast computed tomography and correlation with angiography. Am J Cardiol. 1989;63:870-872.

6. Agatston AS, Janowitz WR, Hildner FJ, Zusmer NR, Viamonte M, Detrano R. Quantification of coronary artery calcium using ultrafast computed tomography. J Am Coll Cardiol. 1990;15:827-832.

7. Raggi P, Callister TQ, Cooil B, et al. Identification of patients at increased risk of first unheralded acute myocardial infarction by electron beam computed tomography. Circulation. 2000;101:850-855.

8. Wong ND, Hsu JC, Detrano RC, Diamond G, Eisenberg H, Gardin JM. Coronary artery calcium evaluation by electron beam computed tomography and its relation to new cardiovascular events. Am J Cardiol. 2000;86:495-498.

9. Arad Y, Spadaro L, Goodman K, Newstein D, Guerci AD. Prediction of coronary events with electron beam computed tomography. $J \mathrm{Am}$ Coll Cardiol. 2000;36:1253-1260.

10. Kondos GT, Hoff JA, Sevrukov A, et al. Electron-beam tomography coronary artery calcium and cardiac events: a 37-month follow-up of 5635 initially asymptomatic low- to intermediate-risk adults. Circulation. 2003;107:2571-2576.

11. Shaw LJ, Raggi P, Schisterman E, Berman DS, Callister TQ. Prognostic value of cardiac risk factors and coronary artery calcium screening for all-cause mortality. Radiology. 2003;228:826-833.

12. Greenland P, LaBree L, Azen SP, Doherty TM, Detrano RC. Coronary artery calcium score combined with Framingham score for risk prediction in asymptomatic individuals. JAMA. 2004;291:210-215.

13. Arad Y, Goodman KJ, Roth M, Newstein D, Guerci AD. Coronary calcification, coronary disease risk factors, C-reactive protein and atherosclerotic cardiovascular disease events: the St Francis Heart Study. J Am Coll Cardiol. 2005;46:158-165.

14. Budoff MJ, Shaw LJ, Liu ST, et al. Long-term prognosis associated with coronary calcium: observations from a registry of 25,253 patients. J Am Coll Cardiol. 2007;49:1860-1870.

15. Schmermund A, Denktas AE, Rumberger JA, et al. Independent and incremental value of coronary artery calcium for predicting the extent of angiographic coronary artery disease: comparison with cardiac risk factors and radionuclide perfusion imaging. J Am Coll Cardiol. 1999; 34:777-786.

16. DeBacker G, Ambrosioni E, Borch-Johnson K, et al. European guidelines on cardiovascular disease prevention in clinical practice. Third joint task force of European and other countries in cardiovascular disease in clinical practice. Eur Heart J. 2003;24:1601-1610.

17. Mieres JH, Shaw LJ, Andrew Arai A, et al. Role of noninvasive testing in the clinical evaluation of women with suspected coronary artery disease: Consensus statement from the Cardiac Imaging Committee, Council on Clinical Cardiology, and the Cardiovascular Imaging and Intervention Committee, Council on Cardiovascular Radiology and Intervention, American Heart Association. Circulation. 2005;111:682-696.

18. Wilson PWF, D'Agostino B, Levy D, Belanger AM, Silbershatz H, Kannel WB. Prediction of coronary heart disease using risk factor categories. Circulation. 1998;97:1837-1847.
19. Grundy SM, Cleeman JI, Merz CNB, et al. Implications of recent clinical trials for the National Cholesterol Education Program Adult Treatment Panel III guidelines. Circulation. 2004;110:227-239.

20. Rifkin RD, Parisi AF, Folland E. Coronary calcification in the diagnosis of coronary artery disease. Am J Cardiol. 1979;44:141-147.

21. McCarthy JH, Palmer FJ. Incidence and significance of coronary artery calcification. Br Heart J. 1974;36:499-506.

22. Rumberger JA, Simons DB, Fitzpatrick LA, Sheedy PF, Schwartz RS. Coronary artery calcium areas by electron-beam computed tomography and coronary atherosclerotic plaque area. A histopathologic correlative study. Circulation. 1995;92:2157-2162.

23. Baumgart D, Schmermund A, Goerge G, et al. Comparison of electron beam computed tomography with intracoronary ultrasound and coronary angiography for detection of coronary atherosclerosis. $\mathrm{J} \mathrm{Am} \mathrm{Coll}$ Cardiol. 1997;30:57-64.

24. Schmermund A, Baumgart D, Gorge G, et al. Coronary artery calcium in acute coronary syndromes: a comparative study of electron beam ct, coronary angiography, and intracoronary ultrasound in survivors of acute myocardial infarction and unstable angina. Circulation. 1997;96: 1461-1469.

25. Rumberger JA, Brundage BH, Rader DJ, Kondos G. Electron beam computed tomographic coronary calcium scanning: a review and guidelines for use in asymptomatic individuals. Mayo Clin Proc. 1999; $74: 243-252$

26. Callister TQ, Cooil B, Raya SP, Lippolis NJ, Russo DJ, Raggi P. Coronary artery disease: improved reproducibility of calcium scoring with an electron-beam CT volumetric method. Radiology. 1998;208:807-814.

27. Hoff JA, Chomka EV, Krainik AJ, Daviglus M, Rich S, Kondos GT. Age and gender distributions of coronary artery calcium detected by electron beam tomography in 35,246 adults. Am J Cardiol. 2001;87: 1335-1339.

28. Schmermund A, Erbel R, Silber S; MUNICH Registry Study Group. Age and gender distribution of coronary artery calcium measured by four-slice computed tomography in 2,030 persons with no symptoms of coronary artery disease. Am J Cardiol. 2002;90:169-173.

29. Budoff MJ, Yang TP, Shavelle RM, Lamont DH, Brundage BH. Ethnic differences in coronary atherosclerosis. J Am Coll Cardiol. 2002;39: $408-412$.

30. Newman AB, Naydeck BL, Whittle J, Sutton-Tyrrell K, Edmundowicz D, Kuller LH. Racial differences in coronary artery calcification in older adults. Arterioscler Thromb Vasc Biol. 2002;22:424-430.

31. Khuran C, Rosenbaum CG, Howard BV, et al. Coronary artery calcification in black women and white women. Am Heart J. 2003;145: 724-729.

32. Jain T, Peshock R, Darren K, et al. African Americans and Caucasians have a similar prevalence of coronary calcium in the Dallas Heart Study. J Am Coll Cardiol. 2004;44:1011-1017.

33. Third Report of the National Cholesterol Education Program (NCEP) Expert Panel on Detection, Evaluation, and Treatment of High Blood Cholesterol in Adults (Adult Treatment Panel III). Final Report. NIH Publication No. 02-5215. 2002 Sep.

34. Detrano R, Guerci AD, Carr JJ, et al. Coronary calcium as a predictor of coronary events in four racial or ethnic groups. N Engl J Med. 2008; 358:1336-1345.

35. Raggi P, Gongora MC, Gopal A, Callister TQ, Budoff M, Shaw LJ. Coronary artery calcium to predict all-cause mortality in elderly men and women. J Am Coll Cardiol. 2008;52:17-23.

36. Grundy SM. Coronary plaque as a replacement for age as a risk factor in global risk assessment. Am J Cardiol. 2001;88(2A):8E-11E.

37. Nasir K, Vasameddy C, Blumenthal RS, Rumberger JA. Comprehensive coronary risk determination in primary prevention: an imaging and clinical based definition combining computed tomographic coronary artery calcium score and national education program risk score. Int $J$ Cardiol. 2006;110:129-136.

38. Ehara S, Kobayashi Y. Yoshiyama M, et al. Spotty calcification typifies the culprit plaque in patients with acute myocardial infarction: an intravascular ultrasound study. Circulation. 2004;110:3424-3429. 
39. Schmermund A, Rumberger JA, Colter JF, Sheedy PF, Schwartz RS. Angiographic correlates of "spotty" coronary artery calcium detected by electron beam computed tomography in patients with normal or near-normal coronary angiograms. Am J Cardiol. 1998;82:508-511.

40. Williams M, Shaw LJ, Raggi P, et al. Prognostic value of number and site of calcified coronary lesions compared with the total score. JACC Cardiovasc Imaging. 2008;1:61-69.

41. Brown ER, Kronmal RA, Bluemke DA, et al. Coronary calcium coverage score: determination, correlates, and predictive accuracy in the multi-ethnic study of atherosclerosis. Radiology. 2008;247:669-675.

42. Tuzcu EM, Kapadia SR, Tutar E, et al. High prevalence of coronary atherosclerosis in asymptomatic teenagers and young adults: evidence from intravascular ultrasound. Circulation. 2001;103:2705-2710.

43. Budoff MJ, Ehrlich J, Hecht HS, Rumberger JR. Letter to the Editor. JAMA. 2004;291:1822.

44. Blaha M, Budoff MJ, Shaw LJ, et al. Absence of coronary artery calcification and all-cause mortality. JACC Cardiovasc Img. 2009;2: 692-700.

45. Greenland P, Smith SC, Grundy SM. Improving coronary heart disease risk assessment in asymptomatic people: role of traditional risk factors and noninvasive cardiovascular tests. Circulation. 2001;104: 1863-1867.

46. O'Rourke RA, Brundage BH, Froelicher VF, et al. American College of Cardiology/American Heart Association expert consensus document on electron-beam computed tomography for the diagnosis and prognosis of coronary artery disease. Circulation. 2000;102:126-140.

47. Expert Panel on Detection, Evaluation, and Treatment of High Blood Cholesterol in Adults. Executive Summary of The Third Report of The National Cholesterol Education Program (NCEP) (Adult Treatment Panel III). JAMA. 2001;285:2486-2497.

48. Pohle K, Ropers D, Mäffert R, et al. Coronary calcifications in young patients with first, unheralded myocardial infarction: a risk factor matched analysis by electron beam tomography. Heart. 2003;89: 625-628

49. Smith SC, Greenland P, Grundy SM. AHA Conference Proceedings. Prevention Conference V: beyond secondary prevention: identifying the high-risk patient for primary prevention. executive summary. Circulation. 2000;101:111-116.

50. Heart Protection Study Collaborative Group. MRC/BHF Heart Protection Study of cholesterol lowering with simvastatin in 20,536 high-risk individuals: a randomized placebo-controlled trial. Lancet. 2002;360:7-22.

51. Raggi P, Cooil B, Callister TQ. Use of electron beam tomography data to develop models for prediction of hard coronary events. Am Heart J. 2001;141:375-382.

52. Raggi P, Shaw LJ, Berman DS, Callister TQ. Prognostic value of coronary artery calcium screening in subjects with and without diabetes. J Am Coll Cardiol. 2004;43:1663-1669.

53. Callister TQ, Raggi P, Cooil B, Lippolis NJ, Russo DJ. Effect of HMG-CoA reductase inhibitors on coronary artery disease as assessed by electron beam computed tomography. $N$ Engl J Med. 1998;339: 1972-1978.

54. Budoff MJ, Lane KL, Bakhsheshi $\mathrm{H}$, et al. Rates of progression of coronary calcium by electron beam tomography. Am J Cardiol. 2000 86:8-11.

Vascular Health and Risk Management

\section{Publish your work in this journal}

Vascular Health and Risk Management is an international, peerreviewed journal of therapeutics and risk management, focusing on concise rapid reporting of clinical studies on the processes involved in the maintenance of vascular health; the monitoring, prevention and treatment of vascular disease and its sequelae; and the involvement of
55. Achenbach S, Ropers D, Pohle K, et al. Influence of lipid-lowering therapy on the progression of coronary artery calcification: a prospective evaluation. Circulation. 2002;106:1077-1082.

56. Shavelle DM, Takasu J, Budoff MJ, et al. HMG CoA reductase inhibitor (statin) and aortic valve calcium. Lancet 2002;359(9312):1125-1126.

57. Raggi P, Cooil B, Shaw L, et al. Progression of coronary calcification on serial electron beam tomography scanning is greater in patients with future myocardial infarction. Am J Cardiol. 2003;92:827-829.

58. Raggi P, Callister TQ, Shaw LJ. Progression of coronary artery calcium and risk of first myocardial infarction in patients receiving cholesterol-lowering therapy. Arterioscler Thromb Vasc Biol. 2004; 24:1272-1277.

59. Hecht HS, Harman SM. Evaluation by electron beam tomography in treated and untreated asymptomatic patients. Am J Cardiol. 2003;91: 1131-1134.

60. Diamond GA, Forester JS. Analysis of probability as an aid in the clinical diagnosis of coronary artery disease. N Engl J Med. 1979;300: 1350-1358.

61. He Z, Hedrick TD, Pratt CM, et al. Severity of coronary calcification by electron beam computed tomography predicts silent myocardial ischemia. Circulation. 2000;101:244-251.

62. Berman DS, Wong ND, Gransar H, et al. Relationship between stressinduced myocardial ischemia and atherosclerosis measured by coronary calcium tomography. J Am Coll Cardiol. 2004;44:923-930.

63. Haberl R, Becker A, Leber A, et al. Correlation of coronary calcification and angiographically documented stenoses in patients with suspected coronary artery disease: results of 1,764 patients. $J$ Am Coll Cardiol. 2001;37:451-457.

64. Nasir K, Kata R, Mao S, et al. Comparison of left ventricular size by computed tomography with magnetic resonance imaging measures of left ventricular mass and volumes: the multi-ethnic study of atherosclerosis. J Cardiovasc Comput Tomogr. 2008;2:141-148.

65. Mao S, Budoff MJ, Oudiz RJ, Bakhsheshi H, Wang S, Brundage BH. A simple single slice method for measurement of left and right ventricular enlargement by electron beam tomography. Int J Card Imaging. 2000; 16:383-390.

66. Lin FY, Devereux RB, Roman MJ, et al. Assessment of the thoracic aorta by multidetector computed tomography: age- and sex-specific reference values in adults without evident cardiovascular disease J Cardiovas Comput Tomogr. 2008;2:298-308.

67. Wajchenberg BL. Subcutaneous and visceral adipose tissue: their relation to the metabolic syndrome. Endocr Rev. 2000;21(6):697-738.

68. Tokunaga K, Matsuzawa Y, Ishikawa K, Tarui S. A novel technique for the determination of body fat by computed tomography. Int J Obes. 1983;7:437-445.

69. Rosito GA, Massaro JM, Hoffmann U, et al. Pericardial fat, visceral abdominal fat, cardiovascular disease risk factors and vascular calcification in a community-based sample: the Framingham Heart Study. Circulation. 2008;117:605-613.

70. Nichols JH, Samy B, Nasir K, et al. Volumetric measurement of pericardial adipose tissue from contrast-enhanced coronary computed tomography angiography: A reproducibility study. J Cardiovasc Comput Tomogr. 2008;2:288-295. 